RESEARCH ARTICLE

\title{
Tigecycline Resistance among Clinical Isolates of Staphylococcus aureus from North-east India
}

\author{
Swati Sharma ${ }^{1 *}$, Deepshikha Bhowmik ${ }^{1 *}$, Shiela Chetri ${ }^{1}$, Deepjyoti Paul ${ }^{1}$, Debadatta Dhar Chanda ${ }^{2}$, Atanu \\ Chakravarty $^{2}$, Amitabha Bhattacharjee ${ }^{1}$ \\ ${ }^{1}$ Department of Microbiology, Assam University, Silchar, Assam, India \\ ${ }^{2}$ Department of Microbiology, Silchar Medical College and Hospital, Silchar, Assam, India \\ ${ }^{*}$ Both the authors should be considered as first author
}

\section{ABSTRACT}

Objective: The current study investigated the resistance against cycline group of antibiotic among clinical isolates of Staphylococcus aureus and molecular characterization with special reference to phenotypic screening of Tigecycline resistance.

Methods: 197 coagulase producing Staphylococcus spp. were collected from private diagnostic laboratories and Silchar Medical College and Hospital, India. Phenotypic screening was done with cycline groups of antimicrobials and genotypically characterized by PCR, targeting coa gene. Detection of tetK, tetM, tet $O$ and tet $X$ genes was performed by PCR. Antibiogram profiling was performed and clonality was also determined.

Results: 127 coagulase positive $S$. aureus were phenotypically screened with cycline group of antibiotics in which prominent resistant type showed highest non-susceptibility against Tigecycline $53.5 \%(n=68)$ while lowest against minocycline $20.5 \%(n=26)$. Multiplex PCR assay showed that tet $(K)$ gene to be the most common cycline group of antimicrobial resistance determinant in $S$. aureus, followed by tet $(M)$ gene. Multiple genes were also observed. However, resistant gene tet( $O$ ) was not reported. Antibiotic susceptibility testing and MIC for therapeutic purpose showed highest susceptibility rate against linezolid. DNA fingerprinting experimented with PFGE had shown seven pulsotypes.

Conclusion: Resistance to cycline group of antibiotics with special reference to Tigecycline is not frequently reported from India. Thus, this study is of clinical interest in terms of selection of proper antimicrobial chemotherapy and also using Tigecycline for phenotypic screening of cycline group of antibiotic resistance in Staphylococcus aureus. $J$ Microbiol Infect Dis 2017; 7(4):173-177

Keywords: Tigecycline, tet genes, Staphylococcus aureus, North-east India

\section{INTRODUCTION}

Tigecycline, a well-tested bacteriostatic drug, acts as an inhibitor of protein synthesis, prevents entry of aminoacyl-tRNA into the A site of the ribosomal RNA [1]. This antibiotic is very active against multidrug resistant bacteria [2,3]. The increasing trends of antibiotic resistance have restricted their clinical usefulness, although the tetracyclines have been used in both human and veterinary medicine [4]. Tetracycline resistance is mediated by efflux pump function and ribosomal protection in Staphylococcus aureus. These resistance mechanisms are acquired from plasmid encoded tet $(K)$ and tet $(\mathrm{L})$ genes and transposon mediated tet( $M)$ and
tet(O) determinants [5]. Currently modern antibiotics are becoming ineffective and difficult to treat severe bacterial infections [6]. Recently $\operatorname{tet}(X)$ gene conferring flavin-dependent monooxygenase activity is responsible for tegicycline resistance [7]. Thus observing the emergence of resistance worldwide the following objective were framed which deals with the screening of cycline group of antimicrobial resistance within clinical isolates of Staphylococcus aureus and molecular characterization of Tigecycline resistance.

\section{METHODS}

Sample collection and identification: 
In total, 197 consecutive non-duplicate, different clinical specimens of Staphylococcus spp. have been collected during the period of one year from April 2014 to March 2015 from Silchar Medical College and Hospital and also from two private diagnostic microbiological laboratories of Silchar, India. The isolates were confirmed by Gram staining, growth on Mannitol Salt Agar and Chrom Agar (HiMedia, Mumbai) and also by coagulase test and latex agglutination test (HiMedia, Mumbai). For molecular characterization of $S$. aureus, PCR was performed targeting coa gene as described earlier [9].

\section{Phenotypic screening of cycline groups of antimicrobial resistance in Staphylococcus aureus:}

Screening of cycline groups of antimicrobial resistance was tested against tetracycline (30 $\mu \mathrm{g})$, doxycycline $(10 \mu \mathrm{g})$, minocycline $(30 \mu \mathrm{g})$ and Tigecycline $(15 \mu \mathrm{g})$ by Kirby Bauer disk diffusion method. Later MRSA was screened by oxacillin and cefoxitin disk diffusion test. The zone dimension was measured and results were interpreted as per Clinical Laboratory Standard Institute (CLSI 2014) [9] guidelines and FDA break point [10].

\section{Molecular characterization of tet genes:}

For genotypic characterization of cycline resistance, multiplex PCR was performed by targeting tet $(K)$, tet $(M)$, tet $(O)$, and $\operatorname{tet}(X)$. The amplification was done by using primers (Table 1) $[11,14,17]$ under the following reaction condition. Initial denaturation was at $95^{\circ} \mathrm{C}$ for 3 min, 30 cycles of $95^{\circ} \mathrm{C}$ for $20 \mathrm{sec}, 50^{\circ} \mathrm{C}$ for 30 $\mathrm{sec}, 72{ }^{\circ} \mathrm{C}$ for $1.2 \mathrm{~min}$ and final extension at 72 ${ }^{\circ} \mathrm{C}$ for $7 \mathrm{~min}$.

\section{Antibiotic susceptibility testing and minimum inhibitory concentration:}

Antibiotic susceptibility against different group of antimicrobials viz: Clindamycin (10 $\mu \mathrm{g})$, Erythromycin $(10 \mu \mathrm{g})$, Pristinomycin $(15 \mu \mathrm{g})$ were tested by disk diffusion methods and Minimum Inhibitory Concentration was done for Oxacillin, Vancomycin, Teicoplanin and Linezolid by agar dilution method and results were noted as per CLSI guidelines [9]. For the study isolates colonies were picked from an overnight culture and were transferred to sterile saline. The suspension was adjusted to 0.5
McFarland standards and incubated on $\mathrm{MH}$ agar plates containing $0.5,1,2,4,8,16,32$ and 64 $\mathrm{mg} / \mathrm{L}$ of the above antibiotics.

\section{DNA fingerprinting by PFGE:}

Typing of isolates, harboring resistance genes was done by Pulsed Field Gel Electrophoresis, using Sma1 restriction enzyme for digestion of bacterial agarose plugs.

\section{RESULTS}

\section{Identification of Staphylococcus aureus:}

A total of 127 isolates were identified as $S$. aureus and further considered for the study.

\section{Phenotypic screening of cycline group of antimicrobials resistance:}

When phenotypically screened for resistance against selected cycline group of antimicrobials, 11 different combinations of antibiogram types were observed. Among them most prominent resistant type showed highest non-susceptibility towards Tigecycline $53.5 \% \quad(n=68)$, followed by tetracycline $41.7 \% \quad(n=53)$, then doxycycline $40.2 \% \quad(n=51)$ and minocycline 20.5\% ( $n=26)$ and the as illustrated in Table 2.

\section{Genotypic characterization of resistance genes:}

When all the combinations of 74 cycline resistant isolates were screened by multiplex PCR assay, thirty three showed different combination of tet genes. Among them tet( $K)$ $(15.0 \%)$ was found to be the most prevalent resistance determinant followed by tet(M) $(7.9 \%)$. The combination of tet(K)/tet(M) (3.1\%) and $\operatorname{tet}(K) / \operatorname{tet}(X)(0.8 \%)$ were also detected and illustrated in Table 3.

\section{Antibiotic susceptibility testing and minimum inhibitory concentration:}

Disc diffusion result showed that out of one hundred twenty seven isolates, 49 (38.6\%) were susceptible to clindamycin, followed by 16 $(12.6 \%)$ to Pristinamycin and 8 isolates $(6.29 \%)$ to erythromycin. While examining minimum inhibitory concentration, Linezolid (81.1\%) and Teicoplanin $(72.4 \%)$ came up with good activity followed by Vancomycin (66.1\%) and Oxacillin (22.8) shown in Table 4.

DNA Fingerprinting of coagulase positive Staphylococcus aureus by PFGE: 
PFGE was performed for clonal dissemination/diversity of coagulase harboring isolates, where seven types of clonal relatedness was observed among the isolates.

Table 1. Primer used for PCR amplification of tet genes.

\begin{tabular}{lcccc}
\hline Primer pairs & Target & Sequence (5'-3') & Amplified product size (bp) & References \\
\hline TetK-F:- & tetK & TTATGGTGGTTGTAGCTAGAAA & 361 & {$[12]$} \\
TetK-R:- & & AAAGGGTTAGAAACTCTTGAAA & & \\
TetM-F:- & tetM & AGT GGA GCG ATT ACA GAA & 159 & {$[12]$} \\
TetM-R:- & & CAT ATG TCC TGG CGT GTC TA & & \\
TetO-F:- & tetO & AATGAAGATTCCGACATTT & 801 & {$[15]$} \\
TetO-R:- & & CTCATGCGTTGTAGTATTCCA & \\
TetX-F:- & tetX $X$ & CAA TAA TTG GTG GTG GAC CC & 468 \\
Tet $X-\mathrm{R}:-$ & & TTC TTA CCT TGG ACA TCC CG & & {$[18]$} \\
\hline
\end{tabular}

Table 2. Phenotypic screening of Cycline resistance.

\begin{tabular}{lc}
\hline Resistant Antibiotic/s & Number of Isolates \\
\hline TGC & 14 \\
TET & 1 \\
TET, DOX & 3 \\
TET, TGC & 3 \\
DOX, TGC & 1 \\
MIN, TGC & 2 \\
TET, DOX, MIN & 2 \\
TET, DOX, TGC & 26 \\
TET, MIN, TGC & 4 \\
DOX, MIN, TGC & 4 \\
TET, DOX, MIN, TGC & 14 \\
OX, CEF & 98 \\
\hline
\end{tabular}

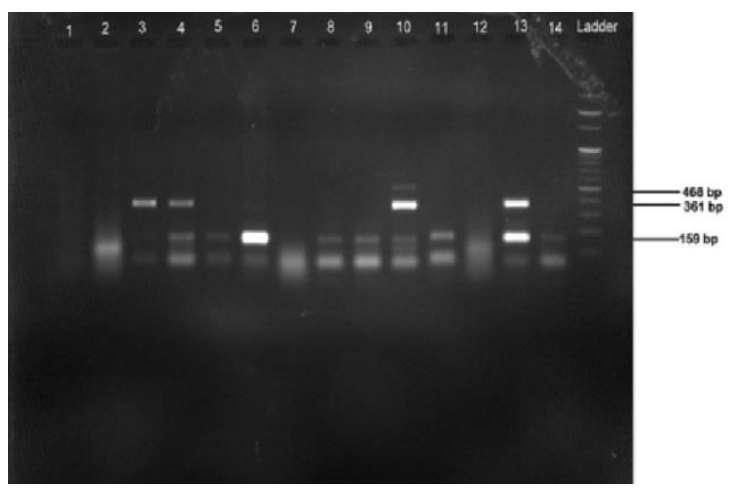

Figure 1. Showing $159 \mathrm{bp}, 361 \mathrm{bp}$ and $468 \mathrm{bp}$ of DNA bands of tet genes in which lane 3,4,5,6,8,9,10,11 \& 13 showing positive result for tet $M$ gene while lane $3,4,10$ and 13 showing positive result for tet $K$ and lane no. 10 showing positive bands for tet $X$ gene respectively, lane 1 showing negative result, lane 14 showing positive control and lane 15 as DNA ladder.

\section{DISCUSSION}

New generation cycline group of antibiotics have been introduced into clinical practice for treatment of Staphylococcus aureus, which is considered to be the most predominant organism among gram positive infections.
Table 3: PCR results of amplified tet genes.

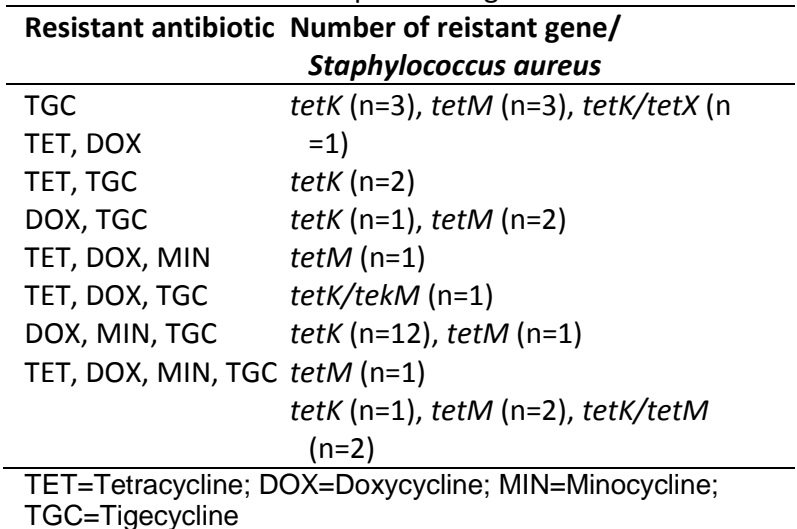

Table 4. Minimum Inhibitory Concentration

\begin{tabular}{lccccccccc}
\hline $\begin{array}{l}\text { Organism/S. aureus } \\
\text { (n=127) (mg/L) }\end{array}$ & 0.5 & 1 & 2 & 4 & 8 & 16 & 32 & 64 & $>64$ \\
\hline Oxacillin & - & - & - & - & 23 & 13 & 20 & 1 & 41 \\
Vancomycin & - & 31 & 13 & 11 & 12 & 8 & 6 & - & 6 \\
Teicoplanin & - & 10 & 6 & 36 & $2 C$ & 15 & 4 & 6 & 10 \\
Linezolid & - & 25 & .0 & 38 & 5 & 4 & 6 & 1 & 8 \\
\hline
\end{tabular}

Continuous exposure of cycline groups of antimicrobials has led to increase in resistant trait. As reported earlier, the treatment option for staphylococcal infection becomes complex due to phenotypic switching, mutational capability and heterogenic resistance [8]. Beside this, patients in critical conditions subjected to broad- 
spectrum antibiotics have life threatening risk by this microorganism as it often exhibit a multidrug resistant phenotype [7]. Thus in order to slow down infection rate the tetracycline and its derivatives doxycycline and minocycline are regarded for promising anti-staphylococcal activity. To add the latest armour in the class, a new Glycylcycline antibiotic, Tigecycline has been introduced into clinical practice in 2005 with greater efficacy [14].

The present study reveals that the prevalence of Tigecycline resistance is highest followed by tetracycline, doxycycline and minocycline, which is in contrast with the observation in UK in 2010 [3].

However, several other studies have reported various resistance determinants of tetracycline. It was reported by Duran et al., 2012 that $41 \%$ and $30.8 \%$ isolates were phenotypically resistant to tetracycline in $S$. aureus and CoNS, in which $42.4 \% S$. aureus isolates harboured both tet(M) and tet $(K)$ genes and $39 \%$ CoNS were also found to carry these genes [11]. A study conducted in Poland in 2000 by Trzcinski et al. observed $66 \%$ tet $(K)$ gene in tetracyclineresistant MRSA [12]. From 1997-1999, 400 MRSA and 200 MSSA isolates were collected from 25 university hospitals in Europe by Schmitz et al. found that 73\% MRSA and $96 \%$ MSSA isolates carry tet(K) gene [13]. In 2005 a study from South Africa reported 58\% (22/38) tet $(K)$ in $S$. aureus from poultry processing plants [14]. Based on their studies it can be assumed that tet $(K)$ gene is more prevalent worldwide and present investigation is also in agreement with the previous reports.

Our study depicts that the isolates carrying tet $(K)$ were resistant to Tigecycline, tetracycline and doxycycline but not to minocycline and those carrying tet $(M)$ were resistant to all the four cycline group of drugs. Whereas the isolates carrying the combination of tet(K)/tet(M) genes, showed resistance to all the four antibiotics but combination of tet $(K) / \operatorname{tet}(X)$ has been reported in only one isolate which showed phenotypic invitro resistance to Tigecycline while tet(O) gene has not been amplified in this study. Therefore, findings of this study are in agreement with an earlier report [15].

Although our study focused on the Tigecycline resistant MRSA, presence of tet(K), tet(M) and combination of tet(K)/tet(M) and tet $(K) / \operatorname{tet}(X)$ were also observed within MSSA isolates. Trzcinski et al., in 2001 reported that tet( $\mathrm{K})$ is responsible for tetracycline resistance in MSSA whereas tet $(M)$ was predominantly present within MRSA strains [12]. In another study tet(M) was contributor for tetracycline and minocycline, while isolates with both tet $(K)$ and tet $(M)$ had a elevated MIC then those with single gene. [14]. Similar finding was too recorded in a previous study of 1990 [15]. Therefore this study suggests that tet $(K)$ confers resistance towards Tigecycline, is most prevalent in this area.

In India, Tigecycline resistance is less frequent among hospital pathogens. Behera et al., 2008 studied the in vitro susceptibility of Tigecycline against hospital isolates [5], the finding of which was in agreement with a study, conducted in Nigeria by Shittu et al., 2011, where all the S. aureus isolates obtained from clinical and nonclinical sources was found to be $100 \%$ susceptible to Tigecycline by disk diffusion method [16]. However there is a conflicting fact in the present study in which most of the isolates showed non-susceptibility towards Tigecycline. In the current study absence of any visible amplification with study primers might be due to heterogeneity of resistance determinants. Linezolid may be considered as therapeutic option as it came up with better efficacy.

In conclusion, with the advent of time the increase in the occurrence of infections caused by Staphylococcus aureus is partially a consequence of inadequate infection control policy and also due to the pathogen's ability to adapt to a changing environment. Thus, this study pointed out that the mechanism of cycline resistance is mainly due to the efflux pumps and ribosomal protection. Presence of tet $(X)$ gene indicates that the bacteria are slowly adapting a newer mechanism of resistance i.e. enzymatic inactivation. During phenotypic screening of cycline group of antimicrobials most of the antibiograms types showed non-susceptibility to Tigecycline and hence this can be used as a screening tool for detection of resistance to this particular group of antimicrobials.

\section{ACKNOWLEDGMENTS}


The authors would like to acknowledge the HOD, Microbiology, Assam University, Silchar and Assam University Biotech Hub for providing infrastructure.

\section{Transparency declarations:}

None

\section{Funding and Sponsorship:}

Nil

\section{Conflicts of Interest:}

None exist.

\section{REFERENCES}

1. Jain $M$, Chakravarti $A$, Verma V, Bhalla $P$. Seroprevalence of hepatitis viruses in patients infected with the human immunodeficiency virus. J Pathol Microbiol 2009; 52:17-19.

2. Brau N. Treatment of chronic hepatitis $\mathrm{C}$ in human immunodeficiency virus/hepatitis $C$ viruscoinfected patients in the era of pegylated interferon and ribavirin. Semin Liver Dis 2005; 25:33-51.

3. Wright TL, Hollander $\mathrm{H}, \mathrm{Pu} \mathrm{X}$, et al. Hepatitis $\mathrm{C}$ in HIV-infected patients with and without AIDS: prevalence and relationship to patient survival. Hepatology 1994; 20:1152-1155.

4. Gerlach JT, Diepolder HM, Jung MC, et al. Recurrence of hepatitis $C$ virus after loss of virusspecific CD4 (+) T-cell response in acute hepatitis C. Gastroenterology 1999; 117:933-941.

5. Antiretroviral therapy for HIV infection in adults and adolescents: Recommendation for a public health approach. World Health Organization 2010 Revision cited 2010 January.

6. Mellor J, Holmes EC, Jarvis LM, Yap PL, Simmonds $P$. Investigation of the pattern of hepatitis $C$ virus sequence diversity in different geographical regions: implications for virus classification. The international HCV Collaborative Study group. J Gen Virol 1995; 76: 2493-2507.

7. Chinchai T, Labout J, Noppornpanth S, et al. Comparative study of different methods to genotype hepatitis $C$ virus type 6 variants. J Virol Methods 2003; 109:195-201.

8. Yang JH, Lai JP, Douglas SD, Metzger D, Zhu $\mathrm{XH}, \mathrm{Ho}$ WZ. Real time RT- PCR for quantitation of hepatitis C virus RNA. J Virol Method 2002; 102:119-128.

9. HIV Sentinel Surveillance and HIV estimation in India 2007: A Technical Brief. [Online]. 2008 [cited 2009 Apr 22]; Available from: URL: http://www.nacoonline.org/upload/Publication/M\&E
\%20Surveillance,\%20Research/HIV\%20Sentinel $\% 20$ Surveillance\%20and\%20HIV\%20Estimation\% 202007_A\%20Technical\%20Brief.pdf

10. Gupta S, Singh S. Hepatitis B and C virus coinfections in human immunodeficiency virus positive north Indian patients. World J Gastroenterol 2006; 12:6879-6883.

11. George SL, Gebhardt J, Klinzman D, et al. Hepatitis C virus viremia in HIV infected individuals with negative $\mathrm{HCV}$ antibody tests. J Acquir Immun Defic Syndr 2002; 31:154-162.

12. Saha ML, Chakrabarty S, Panda $S$, et al. Prevalence of HIV and HBV infection amongst HIV seropositive intravenous drug users and heir noninjecting wives in Manipur, India. Indian $\mathrm{J}$ Med Res 2000; 111:37-39.

13. Saravanan S, Velu V, Kumarasamy N, et al Coinfection of hepatitis $B$ and hepatitis $C$ virus in HIV infected patients in South India. World J Gastroenterol 2007; 13; 5015-5020.

14. Matthews-Greer JM, Caldito GC, Adley SD, et al. Comparision of hepatitis $C$ viral loads in patients with or without Human Immunodeficiency Virus. Clin Diagn Lab Immun 2001; 8: 690-694.

15. Ponamgi S, Rahamathulla S, Kumar $\mathrm{YN}$, et al. Prevalence of hepatitis $\mathrm{C}$ virus (HCV) coinfection in HIV infected individuals in South India and characterization of HCV genotypes. Indian J Med Microbiol 2009; 27:12-16.

16. Karchava M, Sharvadze L, Gatserelia L, Badridze $\mathrm{N}$, Tsertsvadze T. Prevaling HCV genotypes and subtypes among HIV infected patients in Georgia. Georgian Med News 2009; 177:51-55.

17. Pukhrambam PD, Singh NB, Singh LH, Devi KhS. Pattern of hepatitis $\mathrm{C}$ virus genotypes in HIV infected patients in RIMS, Hospital, Imphal, Manipur. J Commun Dis 2007; 39:21-24.

18. Chakravarti A, Dogra G, Verma V, Srivastava AP. Distribution patterns of $\mathrm{HCV}$ genotypes and its association with viral load. Indian J Med Research 2011; 133:326-331.

19. Soriano V, Mocroft A, Rockstorh J, et al. spontaneous viral clearance, viral load and genotype distribution of hepatitis $\mathrm{C}$ virus (HCV) in HIV-infected patients with anti-HCV antibodies in Europe. JID 2008; 198:1337-1344.

20. Reuter S, Oette M, Wilhhelm FC, et al. Prevalence and characteristics of hepatitis $B$ and $C$ virus infections in treatment-naïve HIV-infected patients. Med Microbiol Immunol 2011; 200:39-49. 